\title{
Orientation of One-Dimensional Silicon Polymer Films Studied by X-Ray Absorption Spectroscopy
}

\author{
Md. Abdul Mannan, ${ }^{1,2}$ Yuji Baba, ${ }^{1}$ Tetsuhiro Sekiguchi, ${ }^{1}$ Iwao Shimoyama, ${ }^{1}$ Norie Hirao, ${ }^{1}$ \\ Masamitsu Nagano, ${ }^{2}$ and Hideyuki Noguchi \\ ${ }^{1}$ Quantum Beam Science Directorate, Japan Atomic Energy Agency, Tokai-mura, Naka-gun, Ibaraki 319-1195, Japan \\ ${ }^{2}$ Department of Chemistry and Applied Chemistry, Faculty of Science and Engineering, Saga University, Honjo-1, \\ Saga 840-8502, Japan \\ Correspondence should be addressed to Yuji Baba, baba.yuji@jaea.go.jp
}

Received 28 May 2012; Revised 7 August 2012; Accepted 20 August 2012

Academic Editor: Yanqiu Zhu

Copyright (C) 2012 Md. Abdul Mannan et al. This is an open access article distributed under the Creative Commons Attribution License, which permits unrestricted use, distribution, and reproduction in any medium, provided the original work is properly cited.

\begin{abstract}
Molecular orientations for thin films of one-dimensional silicon polymers grown by vacuum evaporation have been assigned by near-edge X-ray absorption fine structure (NEXAFS) using linearly polarized synchrotron radiation. The polymer investigated was polydimethylsilane (PDMS) which is the simplest stable silicon polymer, and one of the candidate materials for one-dimensional molecular wire. For PDMS films deposited on highly oriented pyrolytic graphite (HOPG), four resonance peaks have been identified in the Si K-edge NEXAFS spectra. Among these peaks, the intensities of the two peaks lower-energy at $1842.0 \mathrm{eV}$ and $1843.2 \mathrm{eV}$ were found to be strongly polarization dependent. The peaks are assigned to the resonance excitations from the Si 1s to $\sigma^{*} \mathrm{p}_{y z}$ and $\sigma^{*} \mathrm{p}_{x}$ orbitals localized at the $\mathrm{Si}-\mathrm{C}$ and $\mathrm{Si}-\mathrm{Si}$ bonds, respectively. Quantitative evaluation of the polarization dependence of the NEXAFS spectra revealed that the molecules are self-assembled on HOPG surface, and the backbones of the PDMS are oriented nearly parallel to the surface. The observed orientation is opposite to the previously observed results for PDMS on the other surfaces such as oxide (indium tin oxide) and metal (polycrystalline copper). The flat-lying feature of PDMS observed only on HOPG surface is attributed to the interaction between CH bonds in PDMS and $\pi$ orbitals in HOPG surface.
\end{abstract}

\section{Introduction}

Polysilanes composed of silicon-silicon backbone have excellent properties in comparison with carbon-based polymers [1]. Polysilanes are considered to be an ideal onedimensional (1D) molecular wire with high electric conductivity since the $\sigma$ electrons in polysilanes are delocalized comparable to those of $\pi$ electrons in the conjugated carbonbased polymers. It has also been predicted that the polysilanes possess electronic structure similar to direct band-gap semiconductor [2].

Among polysilanes, polydimethylsilane (PDMS) is the most fundamental stable silane and considered to be one of the candidate materials in the future nanotechnology with high quality. One of the features of PDMS is the high electric conductivity along the $1 \mathrm{D}$ chain due to the smaller HOMOLUMO band gaps [3]. Thus PDMS and its derivatives are expected to be used as extremely thin wires in molecular electronics. Furthermore, thin films of PDMS possess outstanding physical properties such as photoconductivity and ultraviolet electroluminescence [4-6]. It should be noted that the electric and optical properties in thin films of 1D polymer depend on the configuration of the molecular skeleton. Especially for ultrathin films around monolayer or thinner than monolayer, electric conductivity would become anisotropic depending on the molecular orientation. So it is much important to precisely determine the molecular orientation of polymers on solid surfaces.

It has been found that thin films of silicon polymers synthesized by vacuum evaporation are sometimes self-ordered on solid surfaces. It has been reported that the direction of the backbone of silicon polymers on silicon becomes perpendicular to the surface at high substrate temperature and low evaporation rate $[7,8]$. On the other hand, the direction 
becomes parallel to the surface at low substrate temperature with high evaporation rate [9-12]. In these works, X-ray diffraction was mainly used to determine the structures of the film, so the thickness of the layer is fairly thick in the order of $\mu \mathrm{m}$. These works provide bulk crystal structures rather than molecular orientations in a thin film. Scanning tunnelling microscopy (STM) study confirmed the formation of self-assembled PDMS films of which the silicon backbone chain was perpendicular in respect to the basal plan of graphite substrate [13]. However, STM images gave information about the image of the surface morphology, so the precise titled angle of the PDMS molecules with respect to the backbone configuration and substrate surface has not thoroughly been investigated yet.

In the previous paper, we have investigated the molecular orientation of PDMS films grown on indium tin oxide (ITO) surface which practically used transparent substrate with high electric conductivity. We found that the backbone of the $\mathrm{Si}-\mathrm{Si}$ bond of PDMS molecules is perpendicularly oriented on the ITO surface, and the polymer has a helical conformation rather than zigzag structure with polar angle around $40^{\circ}$ [14].

In this paper, we present the results for the orientation of PDMS films grown on highly oriented pyrolytic graphite (HOPG) substrate which is one of the ideal materials with completely flat and inert surface. The results are compared with the previously obtained ones on the different substrates, and the origin of the difference in the orientation is discussed.

\section{Experimental Method}

2.1. Experimental Setup. All the experiments were performed in situ at the end station of beam line 27A (BL-27A) of the Photon Factory, High Energy Accelerator Research Organization (KEK-PF). Details of the BL-27A were reported elsewhere [15]. Briefly, double crystals of In $\mathrm{Sb}$ (111) were used as a monochromator. The total energy resolution of this monochromator was $0.90 \mathrm{eV}$ at $1.84 \mathrm{keV}$ (Si K-edge). The synchrotron radiation (SR) beam was generated from the bending magnet, and it was linearly polarized in the horizontal direction. The typical photon flux was $10^{10}$ photons $\cdot \mathrm{cm}^{-2} \cdot \mathrm{s}^{-1}$.

The end station of the BL-27A has two ultrahigh vacuum chambers: (i) a main chamber for X-ray photoelectron spectroscopy (XPS) and NEXAFS measurements and (ii) a preparation chamber for vacuum evaporation. The base pressure of both the chambers was in the order of $1 \times$ $10^{-8} \mathrm{~Pa}$. The main chamber consisted of a five-axis manipulator, a hemispherical electron energy analyzer (VSW Co. Class-100) for XPS measurements. In the preparation chamber, a vacuum evaporator and a sample transfer systems were installed. For XPS measurements, the X-rays were irradiated at 55 degree from surface normal, and a take-off direction of photoelectrons was surface normal.

The vacuum evaporator consisted of a quartz crucible surrounded by spiral-type tungsten filament. The crucible was floated at $+1.5 \mathrm{kV}$ while the filament was grounded.
Therefore, the crucible was heated by the electron bombardment. The distance between the crucible and the substrate was $15 \mathrm{~cm}$. A shutter electrically isolated from the ground was equipped with it in order to control the evaporation rate of the source material. Since some of the evaporated molecules are ionized due to the surface ionization, a positive current was observed at the shutter. Thickness of the films was precisely determined by the product of the shutter current and the evaporation time which was calibrated by XPS measurements.

2.2. Film Synthesis. The source powder material purchased from GELEST Inc. was polydimethylsilane (PDMS) $\left[\mathrm{Si}\left(\mathrm{CH}_{3}\right)_{2}\right]_{n}$. The average molecular weight of the PDMS is 2000 so the number of silicon atoms in a PDMS molecule was about 28. Fresh surface of the HOPG was obtained by cleaving in atmosphere, and it was attached on a sample holder by carbon tape and then immediately transferred into the preparation chamber.

The PDMS powder was put in the quartz crucible where the crucible was heated by the bombardment of $1.5 \mathrm{keV}$ electrons from the backside. It was confirmed that the evaporation temperature of the crucible measured by a thermocouple was $250^{\circ} \mathrm{C}$. The evaporation was performed for $5 \mathrm{~min}$ to $15 \mathrm{~min}$ at the working pressure of $2.2 \times 10^{-5} \mathrm{~Pa}$. The filament current was $4.1 \mathrm{~A}$, whereas the shutter current was $500 \mathrm{pA}$. The substrate was kept at room temperature during deposition. We have measured the Si 1 s and $\mathrm{C} 1 \mathrm{~s}$ peaks in the XPS spectrum for the deposited samples, and then the thickness was calculated by the peak intensity ratio using photoionization cross-section [16] and inelastic mean free-paths (IMFP's) [17] (see Section 3.1).

The electronic structure and molecular orientation were investigated by XPS and NEXAFS measurements. The NEXAFS spectra were taken with the total electron yield mode by recording the sample current. The total electron yields were normalized by the photon flux that was monitored by the current of aluminium foil located in front of the sample. The sample was horizontally located, and it was rotated around the vertical axis for the measurements of the polarization dependences.

Irradiation of intense X-rays on organic molecules would sometimes induce the decomposition. In order to check the radiation-induced decomposition, we have irradiated the PDMS films by SR X-rays for one day (total photon fluence was about $10^{15}$ photons $\cdot \mathrm{cm}^{-2}$ ). However, neither XPS nor NEXAFS spectra changed probably because of the low photoabsorption cross-sections of silicon and carbon (in the order of $10^{4}$ barn). So we can ignore the radiation-induced decomposition of molecules in the present experiments.

\section{Results and Discussion}

3.1. Electronic Structure and Film Thickness by XPS Analysis. Figure 1 shows XPS wide scan spectra for the PDMS films with two different deposition times. The XP spectrum for a clean HOPG surface before the deposition is also shown as a 


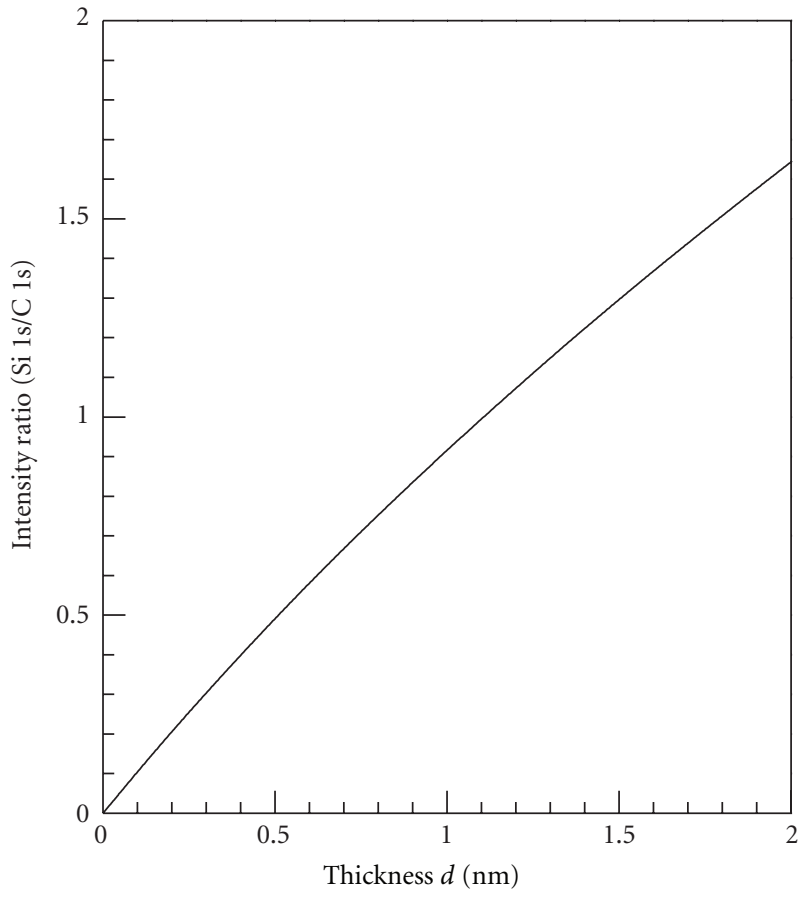

(a)

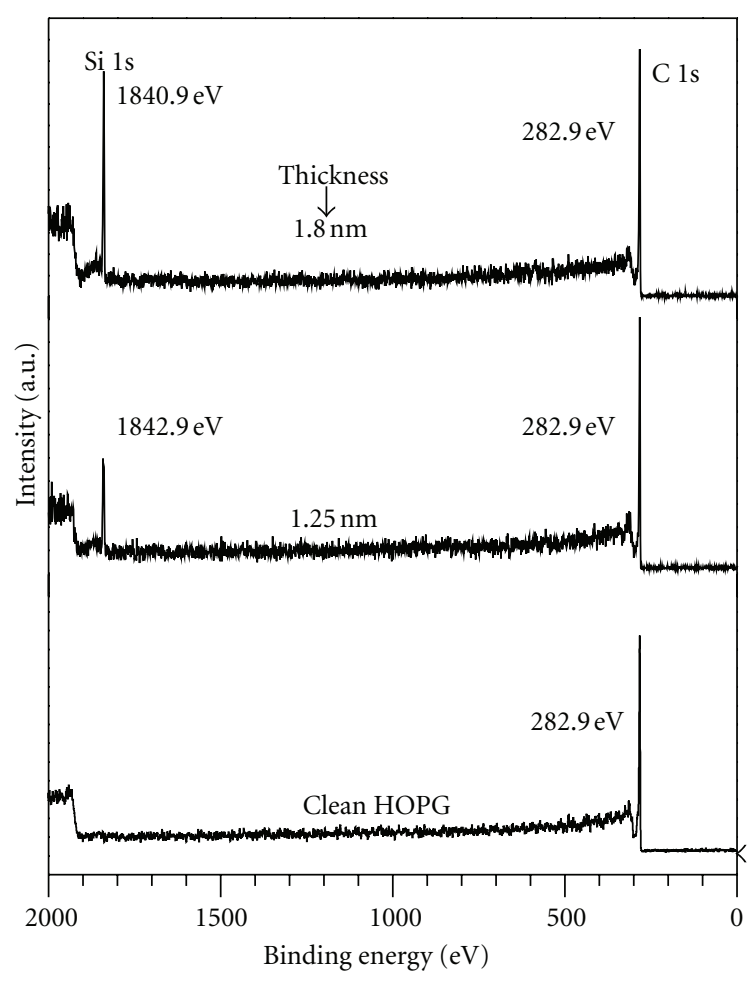

(b)

FIGURE 1: XPS wide scan spectra for the PDMS films with different thickness evaporated on HOPG surface at ambient temperature. The sample surface was irradiated with the SR beam of $2.2 \mathrm{keV}$ photons as an excitation source. The spectra were taken at the step energy of $1.0 \mathrm{eV}$. The relationship between the film thickness and the intensity ratio of $\mathrm{Si} 1 \mathrm{~s} / \mathrm{C} 1 \mathrm{~s}$ calculated by (1) is (a).

bottom spectrum. First, the thicknesses of the PDMS films is determined on the basis of the XPS peak intensities.

The intensities of Si 1s photoelectrons $\left(I_{\mathrm{Si}}\right.$ ss $)$ and $\mathrm{C} 1 \mathrm{~s}$ photoelectrons $\left(I_{\mathrm{C}}\right.$ ss $)$ were calculated by using the following two equations [18] supposing that the film is homogeneous:

$$
\begin{aligned}
I_{\mathrm{Si} 1 \mathrm{~s}}= & K \sigma_{\mathrm{Si} 1 \mathrm{~s}} \times \lambda_{\mathrm{Si} 1 \mathrm{~s} \text { in PDMS }} \times n_{\mathrm{Si} \text { 1s in PDMS }} \\
& \times\left[1-\exp \left(\frac{-d}{\lambda_{\mathrm{Si} 1 \mathrm{~s} \text { in PDMS }}}\right)\right], \\
I_{\mathrm{C} 1 \mathrm{~s}}= & K \sigma_{\mathrm{C} 1 \mathrm{~s}} \times \lambda_{\mathrm{C} 1 \mathrm{~s} \text { in } \mathrm{HOPG}} \times n_{\mathrm{C} \text { in } \mathrm{HOPG}} \\
& \times \exp \left(\frac{-d}{\lambda_{\mathrm{C} 1 \mathrm{~s} \text { in PDMS }}}\right)+K \sigma_{\mathrm{C} \text { 1s }} \times \lambda_{\mathrm{C} \text { 1s in PDMS }} \\
& \times n_{\mathrm{C} \text { in PDMS }} \times\left[1-\exp \left(\frac{-d}{\lambda_{\mathrm{C} \text { 1s in PDMS }}}\right)\right],
\end{aligned}
$$

where $K$ is the constant depending on detection efficiency of the electron energy analyser, $\sigma$ (barn) is photoionization cross-section, $\lambda(\mathrm{nm})$ is IMFP of the photoelectrons of the respective elements, $n$ is the atomic concentration of the element in the material indicated as subscripts, and $d(\mathrm{~nm})$ is the thickness of the PDMS film. The values used for the calculation of $\sigma$ for $\mathrm{Si} 1 \mathrm{~s}$ and $\mathrm{C} 1 \mathrm{~s}$ at $2.2 \mathrm{keV}$ photons were $1.02 \times 10^{5}$ and $5.0 \times 10^{3}$ barns, respectively [16]. The Figure 1(a) shows the relationship between the thickness $d$ $(\mathrm{nm})$ and the peak intensity ratio of $\mathrm{Si} 1 \mathrm{~s} / \mathrm{C}$ 1s. From the experimentally obtained $\mathrm{Si}$ 1s/C 1s values, the thicknesses of the PDMS films for two samples are determined to be $1.25 \mathrm{~nm}$ (middle spectrum) and $1.80 \mathrm{~nm}$ (upper spectrum).

Figure 2 shows the Si 1s XPS narrow scan for the samples with the different thickness. The sample surface was excited by the SR beam of $2.2 \mathrm{keV}$ photons, and the spectra were taken at the step energy of $0.07 \mathrm{eV}$. Two sharp peaks at the binding energy of $1842.9 \mathrm{eV}$ and $1840.7 \mathrm{eV}$ are observed for the thin sample with the thickness of $1.25 \mathrm{~nm}$. The intensity of the peak observed at $1842.9 \mathrm{eV}$ becomes significantly low with the thickness (sample with the thickness of $1.80 \mathrm{~nm}$ ). The energy of the main peak at $1840.7 \mathrm{eV}$ is the same as the previously reported values for multilayered PDMS films [14]. Also the binding energies of the $\mathrm{C} 1 \mathrm{~s}$ peak for these two layers are located around $282.8 \mathrm{eV}$, which is similar to those for the $\mathrm{SiC}$ layer [19-21]. Thus the main peak observed at $1840.7 \mathrm{eV}$ is surely attributed to the silicon atoms in PDMS molecules. One of the biggest questions is that why two peaks are found in the Si 1s spectra. We confirmed that the peak splitting is not due to the decomposition of PDMS by X-ray irradiation because the peak structures have never changed even after long-time irradiation. It was reported that the peaks for the low-dimensional Si always stay at the higher binding energy region than that for the bulk-Si $[22,23]$. We consider that low-dimensional feature for thin film might be one of the reasons for the peak found at $1842.9 \mathrm{eV}$ [23]. So the possible explanations are that (i) the peak energy at $1842.9 \mathrm{eV}$ is due 


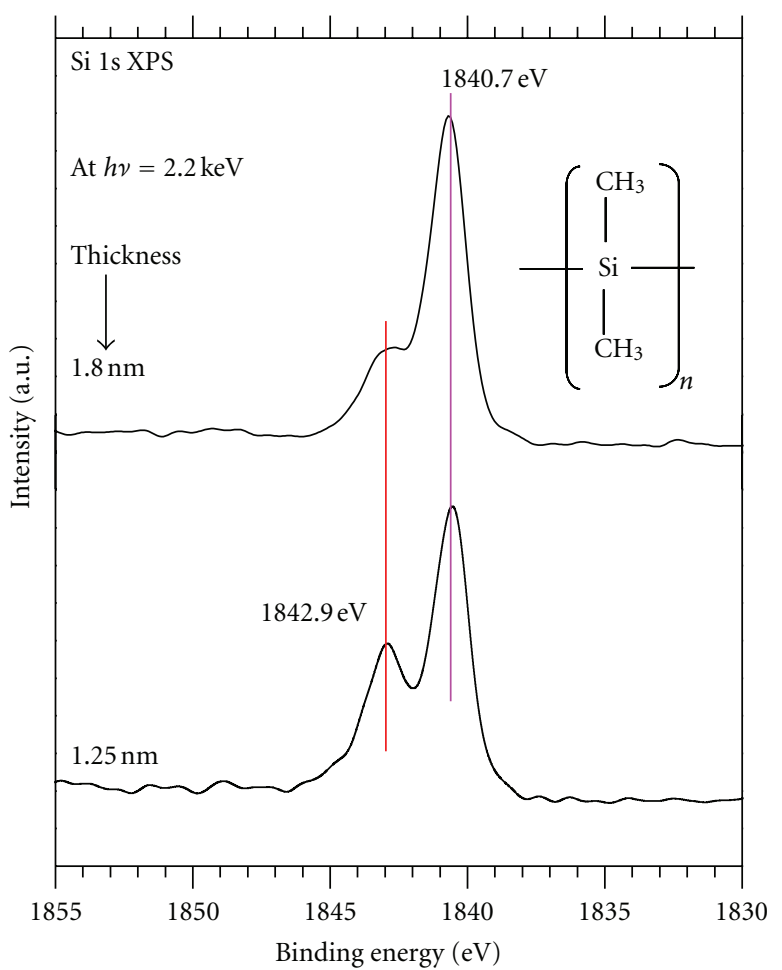

Figure 2: Si 1s narrow scan XP spectra for the PDMS films evaporated on HOPG surface with different thickness. The molecular formula of the PDMS is also shown in the figure in which the molecular weight is around 2000, and the number of silicon atoms $(n)$ is about 28 . The sample surface was excited by the SR beam of $2.2 \mathrm{keV}$ photons as an excitation source and the spectra were taken at the step energy of $0.07 \mathrm{eV}$.

to the formation of monolayer and (ii) the peak energy at $1840.7 \mathrm{eV}$ is due to the formation of multilayer of the PDMS molecules.

Since XPS could not provide information regarding molecular orientation of chemical bonds, NEXAFS has been applied in order to elucidate molecular orientation and quantitative evaluation of polar angle with respect to the backbone configuration of PDMS in the subsequent section.

3.2. Quantitative Evaluation of Molecular Orientation by Si K-Edge NEXAFS Analyses. Figures 3(a) and 3(b) show Si $K$-edge NEXAFS spectra for the PDMS films with different thickness. The spectra were recorded at different incidence angles $\theta$ of the SR beam. In the spectra, polarization dependence of NEXAFS has been clearly observed for both samples. At the nearly normal incidence $\left(\theta=80^{\circ}\right)$ of the SR, four peaks marked by A, B, C, and D are observed. The photon energies of the four peaks are located at 1842.0, $1843.2,1845.2$, and $1848.6 \mathrm{eV}$, all of which are found to be the same as those observed for the samples deposited on ITO surface [14].

Among the four peaks, the peaks A and B are strongly polarization dependent. The intensity of the peak $A$ is increased while the intensity of peak B is decreased at the small incidence angle of the SR beam $\left(\theta=10^{\circ}\right)$. For comparison, Si $K$-edge NEXAFS spectra for the PDMS films on ITO surface with different thickness are shown in Figure 4 [14]. It has been observed that the intensity of peak $A$ decreased and the intensity of peak $B$ increased when the incident angle becomes low $\left(\theta=10^{\circ}\right)$. In the previous paper, we have concluded that the backbone of the PDMS, that is, the $\mathrm{Si}-\mathrm{Si}$ bond is perpendicularly oriented on the ITO surface [14]. The present result for the polarization dependence of PDMS on HOPG is really opposite to that of PDMS on ITO. The result suggests that the $\mathrm{Si}-\mathrm{Si}$ bond is parallel to the HOPG surface.

Let us go back to Figure 3. As to the peaks in Si Kedge NEXAFS spectra for $\mathrm{Si}$ polymer, the origin of the resonance peaks for PDMS or similar compounds has been experimentally and theoretically assigned $[3,24]$. According to McCrary et al., the low-energy resonance peak $\mathrm{A}$ at $1842.0 \mathrm{eV}$ is due to the resonance excitation from the $\mathrm{Si} 1 \mathrm{~s}$ to $\sigma^{*} \mathrm{p}_{y z}$ orbital localized at the Si-C bond, and the high-energy resonance peak $\mathrm{B}$ at $1843.2 \mathrm{eV}$ is attributed to the resonance excitation from the Si 1 s to $\sigma^{*} \mathrm{p}_{x}$ orbital along the Si-Si bond [24]. Since higher-energy peaks C and D are not apparently polarization dependent, we shall hereafter concentrate on the polarization dependences of the peaks A and B.

To our knowledge, there have been no experimental works for precise determination of the angle for silicon polymer films on solid surface. Concerning this fact, it is very important to elucidate the exact polar angle of the PDMS molecule. As seen, a remarkable feature observed in the peaks A and B is the clear polarization dependence of their intensity. The peak intensity I for the NEXAFS spectra using SR beam of the electric field $\mathbf{E}$ could be expressed by the following equation [25]:

$$
\mathbf{I} \propto|\mathbf{E} \cdot \mathbf{O}|^{2} \propto \cos ^{2} \delta
$$

where $\mathbf{O}$ is the direction of the final state orbital, and $\delta$ is the angle between $\mathbf{E}$ and $\mathbf{O}$. Therefore, considering the polarization dependences as found in the Figure 3, we can suppose that the final state orbitals represented by the peak $\mathrm{A}$ are perpendicular while those represented by the peak B are parallel to the basal plane of the HOPG. In this viewpoint, the $\sigma^{*}$ orbitals localized at the Si-C bond (hereafter we denote $\sigma^{*}{ }_{\mathrm{Si}-\mathrm{C}}$ ) are almost perpendicular, and the $\sigma^{*}$ orbitals localized at the Si-Si bond (hereafter we denote $\sigma^{*}{ }_{\mathrm{Si}-\mathrm{Si}}$ ) are parallel to the HOPG substrate surface.

In order to get more quantitative clarification of the tilted angles, we estimate the angles of $\sigma^{*}{ }_{\mathrm{Si}-\mathrm{C}}$ and $\sigma^{*} \mathrm{Si}-\mathrm{Si}$ with respect to the silicon backbone configuration and the substrate surface. In that case, the resonance intensity in the NEXAFS spectrum could be simplified by the following equation:

$$
\mathrm{I}(\theta)=\mathrm{A}\left[\mathrm{P} \cdot \mathrm{I}_{\mathrm{p}}+(1-\mathrm{P}) \cdot \mathrm{I}_{\mathrm{v}}\right]
$$

where $I_{p}$ and $I_{v}$ are the transition intensities associated with the angle-dependent matrix elements parallel and vertical to the electric field vector, respectively, $\mathrm{A}$ is the normalization, and $\mathrm{P}$ is the polarization factor [25]. The polarization factor of the SR beam under the present experimental condition was estimated to be about 0.95. The PDMS molecule on 


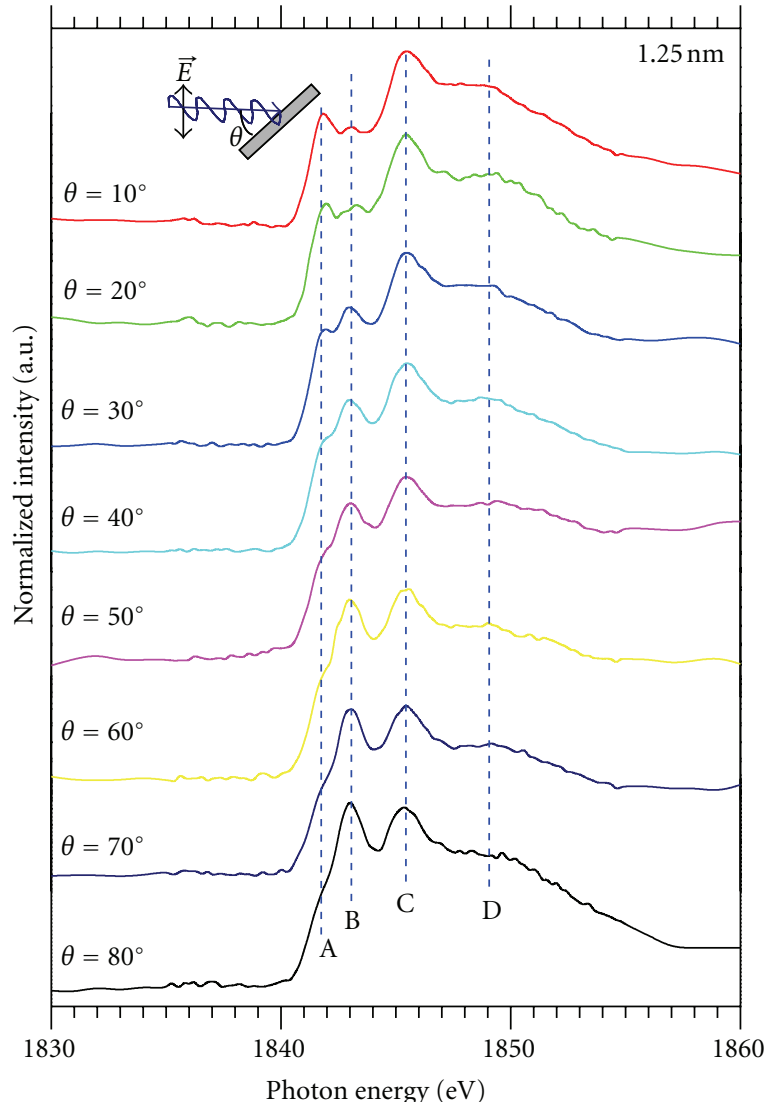

(a)

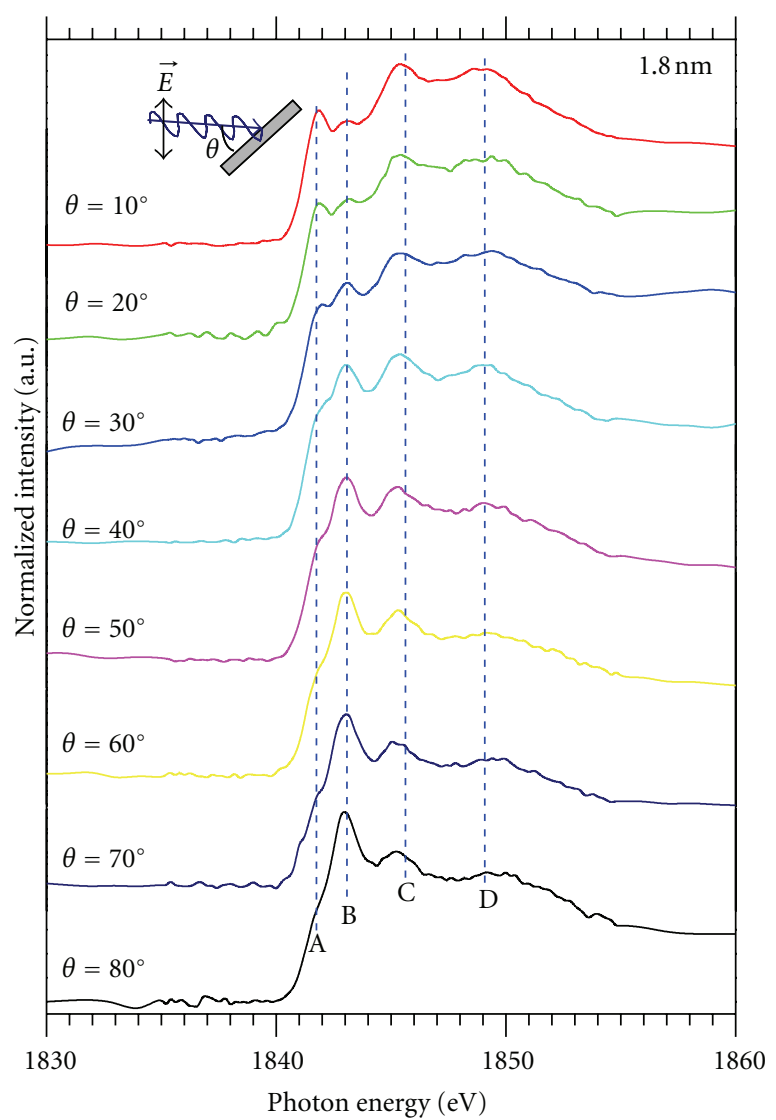

(b)

FIGURE 3: Si K-edge NEXAFS spectra for the samples deposited on HOPG substrate with different thickness: (a) $1.25 \mathrm{~nm}$ and (b) $1.80 \mathrm{~nm}$. The NEXAFS spectra were recorded by the total electron yield mode at the different incident angles $\theta$ of the SR beam. Definition of the incidence angle $\theta$ is shown inside of this figure.

the HOPG surface has symmetry higher than threefold, so the intensities can be simply expressed by the following four equations: in the case of vector type orbital, the equation becomes

$$
\begin{gathered}
\mathrm{I}_{\mathrm{v}}^{\|}=\left(\frac{1}{3}\right) \cdot\left[1+\left(\frac{1}{2}\right) \cdot\left(3 \cos ^{2} \theta-1\right) \cdot\left(3 \cos ^{2} \alpha-1\right)\right], \\
\mathrm{I}_{\mathrm{v}}^{\perp}=\left(\frac{1}{2}\right) \cdot \sin ^{2} \alpha .
\end{gathered}
$$

In the case of plane type orbital, the equation becomes

$$
\begin{gathered}
\mathrm{I}_{\mathrm{p}}^{\|}=\left(\frac{2}{3}\right) \cdot\left[1-\left(\frac{1}{4}\right) \cdot\left(3 \cos ^{2} \theta-1\right) \cdot\left(3 \cos ^{2} \gamma-1\right)\right], \\
\mathrm{I}_{\mathrm{p}}^{\perp}=\left(\frac{1}{2}\right) \cdot\left[1+\cos ^{2} \gamma\right],
\end{gathered}
$$

where $\alpha$ is the polar angle, that is, the angle between the surface normal and the vector-type $\sigma^{*}{ }_{\mathrm{Si} \text {-Si }}$ orbital (peak B). It should be noted that $\alpha$ is the same as the angle between surface and the Si-Si bond. $\gamma$ is also the polar angle that is, the angle between the surface normal and the normal direction of the plane-type $\sigma^{*} \mathrm{Si-C}$ orbital (peak A). The value of $\gamma$ is the same as the angle between surface and $\mathrm{Si}-\mathrm{C}$ bond. The definition of the polar angles $\alpha$ and $\gamma$ is shown in the Figure 5 .
Figures 6(a) and 6(b) show the normalized peak intensity I of the peaks A and B of the NEXAFS spectra as a function of the incident angle $(\theta)$ of the SR beam. The details of the normalization procedure have been described in the caption of Figure 6. The spectrum taken at the small incidence angle $\left(\theta=10^{\circ}\right)$ shows broad feature. It is seen that the intensity of the peak A decreased, while the intensity of the peak B increased when the spectra were taken at the incidence angle $\theta=80^{\circ}$ (See Figure 3). Therefore, the areas of the peaks A and $B$ found strongly polarization dependent are calculated by peak deconvolution using Gaussian-curve fitting technique. The normalized intensities of the peaks A and B are plotted as filled circles with solid line which are shown in Figure 6.

It has been observed that the experimental data for both the samples almost follow the line with the polar angle $\sim 65^{\circ}$. We already discussed the standing-up orientation in more detail on the basis of the zigzag and helical conformation of the PDMS films grown on ITO surface [14]. We proposed that the multilayer film composed of helix structure was perpendicularly oriented on the ITO surface with the polar angle of around $40^{\circ}$. Present results confirmed that the experimental values of the polar angle for both the vector and plane type orbitals follow the theoretical line around $65^{\circ}$ (See Figures 6(a) and 6(b)). 


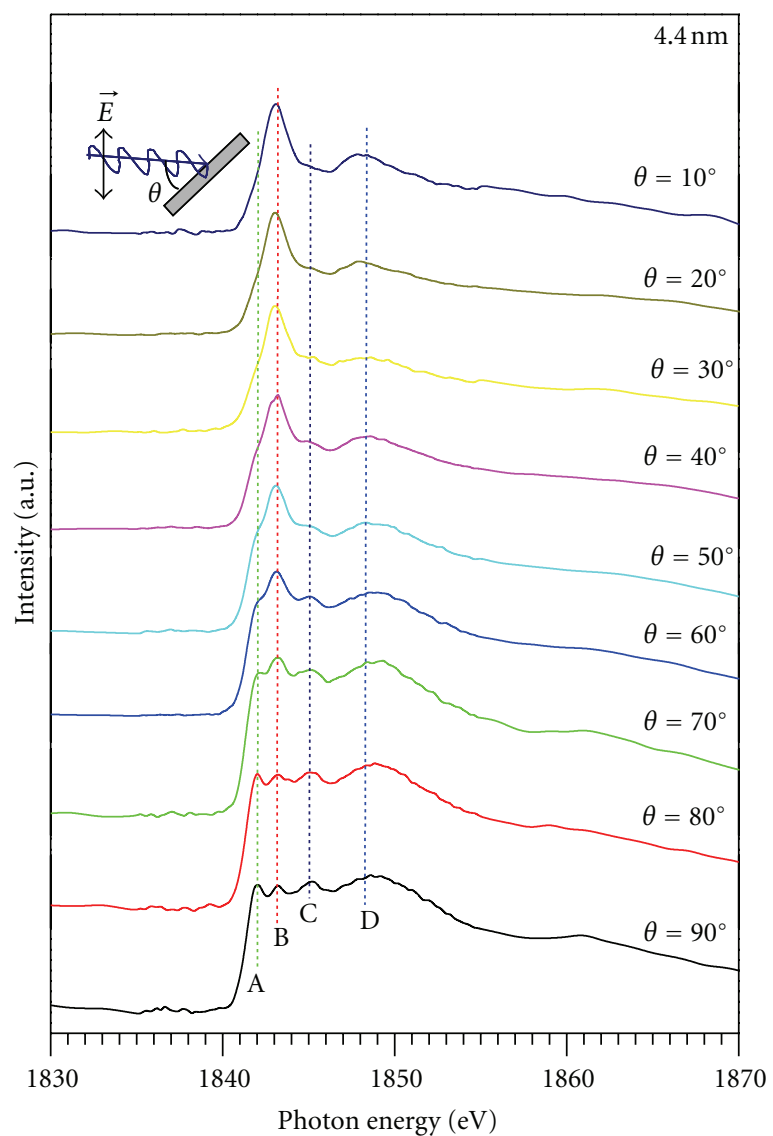

(a)

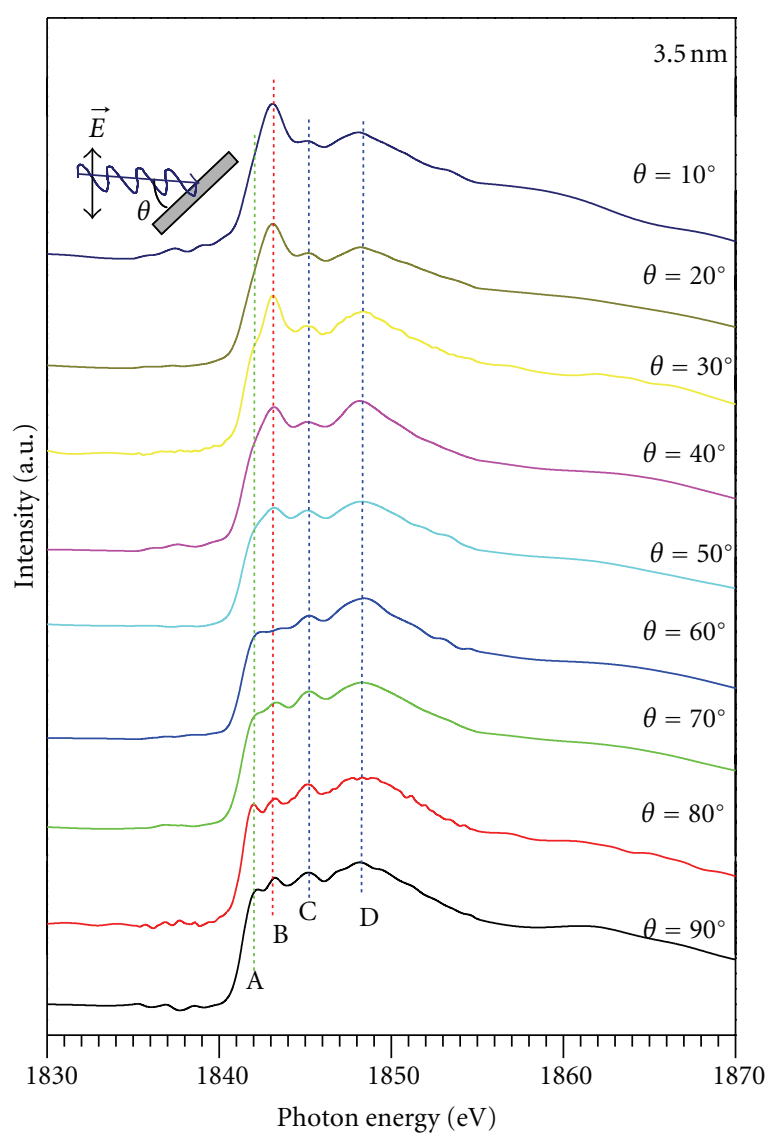

(b)

FIGURE 4: Si K-edge NEXAFS spectra for the samples deposited on ITO substrate with different thickness: (a) $4.4 \mathrm{~nm}$ and (b) $3.5 \mathrm{~nm}$ [14]. The NEXAFS spectra were recorded by the total electron yield mode at the different incident angles $\theta$ of the SR beam. Definition of the incidence angle $\theta$ is shown inside of this figure.

If the structure is flat-lying helical, $\alpha$ equals 48.5 degree, and $\gamma$ equals 41.5 degree. These angles are just complementally angles of those of the standing-up helical configuration. On the other hand, for the flat-lying zigzag configuration, there are roughly two cases. One is that the $\mathrm{Si}-\mathrm{C}$ plane is perpendicular to the surface. In this case, $\mathrm{Si}$ atoms locate close and far from the surface one by one. For this configuration, the $\alpha$ equals $54.5^{\circ}$ and $\gamma$ equals $0^{\circ}$. The second case is that the $\mathrm{Si}-\mathrm{Si}$ bond is parallel to the surface. In this case, all of the Si atoms are close to the surface. For this configuration, $\alpha$ equals $90^{\circ}$ and $\gamma$ equals $0^{\circ}$.

The experimental data show that $\alpha$ equals $65^{\circ}$, and $\gamma$ equals $65^{\circ}$. It is wonder that the values are not exactly the same as those of the flat-lying helical structure. But if the configuration is flat-lying zigzag, then at least $\gamma$ value should be nearly 0 for both the cases. So we can suppose that the configuration is flat-lying helical structure with the polar angle of around $65^{\circ}$.

A question might be raised as why the orientations of the PDMS molecules are opposite between HOPG and ITO surfaces. At present, we cannot give a definite answer to this interesting phenomenon. One plausible speculation about standing-up configuration is as follows.
We have also investigated the orientation of PDMS on other substrates such as polycrystalline copper, and it was found that the backbones are perpendicular to the surface. As to the flatness of the surface, we observed that the ITO surface is vertically undulated in the order of $10 \mathrm{~nm}$ [26]. The surface of copper seems to be also undulated in larger scale. Therefore, the first layer of PDMS on the ITO and $\mathrm{Cu}$ surfaces cannot lie down due to the stress in the molecular backbone. This is the reason why the PDMS molecules stand up on a rough surface. On the other hand, HOPG has perfectly flat surface without defects and steps, so the first layer of PDMS can lie flat along the surface. It is considered that the succeeding over layers follows the orientation of the first layer.

As to the question why PDMS molecules lie flat only on HOPG surface, one plausible explanation is the relatively strong interaction between $\mathrm{CH}$ bonds in PDMS and $\pi$ orbitals $(\mathrm{CH} / \pi$ interaction) at the HOPG surface. The $\mathrm{CH} / \pi$ interaction is a kind of hydrogen bond operating between a soft acid $\mathrm{CH}$ and a soft base $\pi$-system like not only benzenes but graphites, fullerenes, and nanotubes. Nishio et al. have reported that the $\mathrm{CH} / \pi$ interaction exists as ubiquitous force and stressed the importance of the $\mathrm{CH} / \pi$ interaction in 


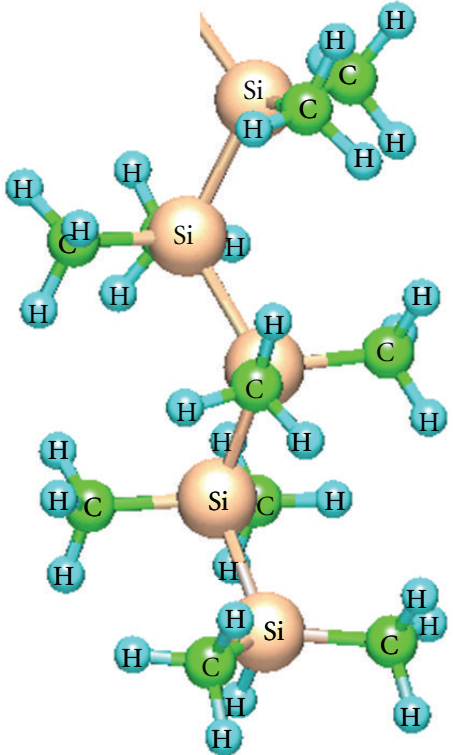

(a)

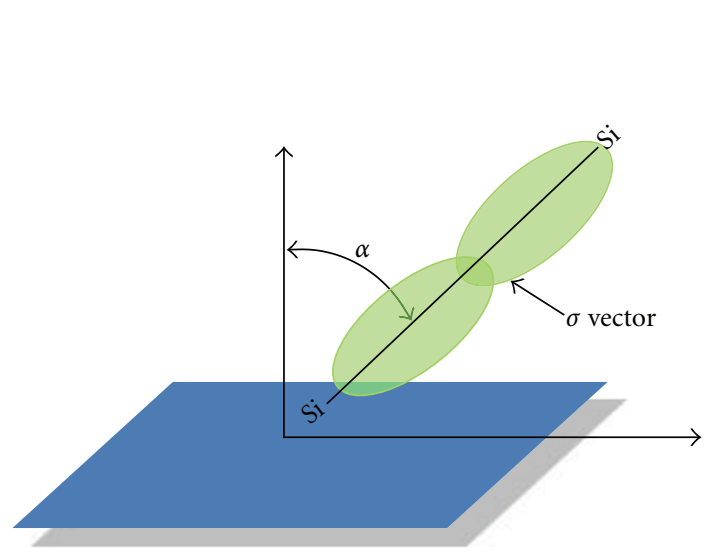

(c)

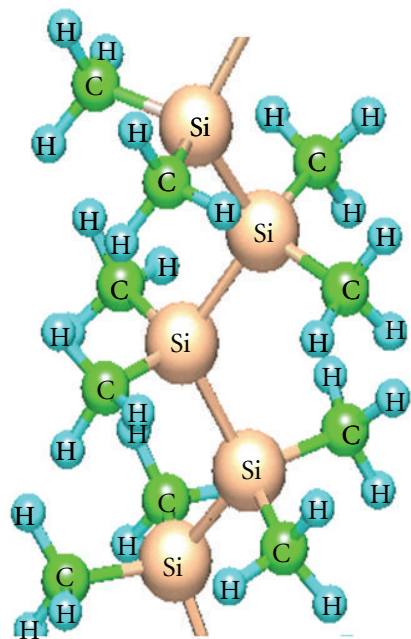

(b)

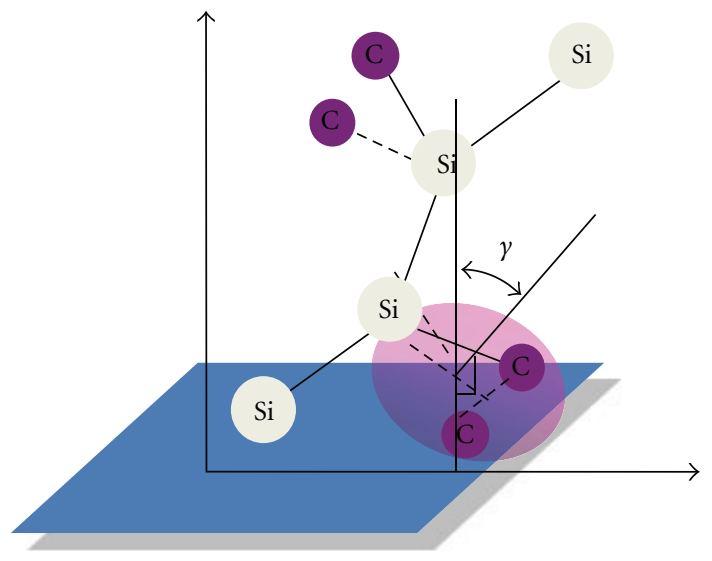

(d)

FIGURE 5: Sketch of the PDMS molecule in which only five silicon atoms are considered: (a) zigzag configuration, (b) helical configuration, (c) definition of the polar angle $\alpha$ in respect to the $\mathrm{Si}-\mathrm{Si} \sigma$ vector, and (c) definition of the polar angle $\gamma$ in respect to the Si-C plane. The structures (a) and (b) are sketched by the WinMOPAC3.9 version of the computer program.

various fields of chemistry [27-29]. As to the solid surface, Linares et al. reported the configuration of a porphyrin derivatives on graphite by scanning tunneling microscopy and molecular simulation, and they have shown that methyl groups point toward the surface of graphite, suggesting the occurrence of $\mathrm{CH} / \pi$ hydrogen bonds [30]. In the present case, a PDMS molecule has six $\mathrm{CH}$ bonds in each silicon atom, and one of the $\mathrm{CH}$ bonds would contribute to bind with HOPG surface through the $\mathrm{CH} / \pi$ interaction.

In summary, we have investigated the molecular configuration of PDMS films deposited on HOPG surface and demonstrated that the molecular skeleton of the polymer is just parallel to the basal plane of HOPG. More detailed analysis revealed that the PDMS molecules lie flat on the HOPG surface showing helical structure with the polar angle of around $65^{\circ}$. Since polysilanes composed of siliconsilicon backbone are one of the candidate materials as $1 \mathrm{D}$ molecular wire with high electric conductivity, the present results will shed light on the future applications of polysilanes to molecular electronic devices with high quality.

\section{Conclusions}

We have analysed the self-assembling, electronic structure, and molecular orientation of 1D PDMS films by XPS and NEXAFS spectroscopy using linearly polarized synchrotron radiation as an excitation source. XPS confirmed the formation of monolayer and multilayer films in the samples. In the Si $K$-edge NEXAFS, four resonance peaks are identified of which two peaks are found to be strongly polarization dependent. On the basis of the polarization dependence of NEXAFS, finally, we found that the backbones of the PDMS molecules are nearly parallel in respect to the HOPG surface. The results are just opposite to those for our previous 


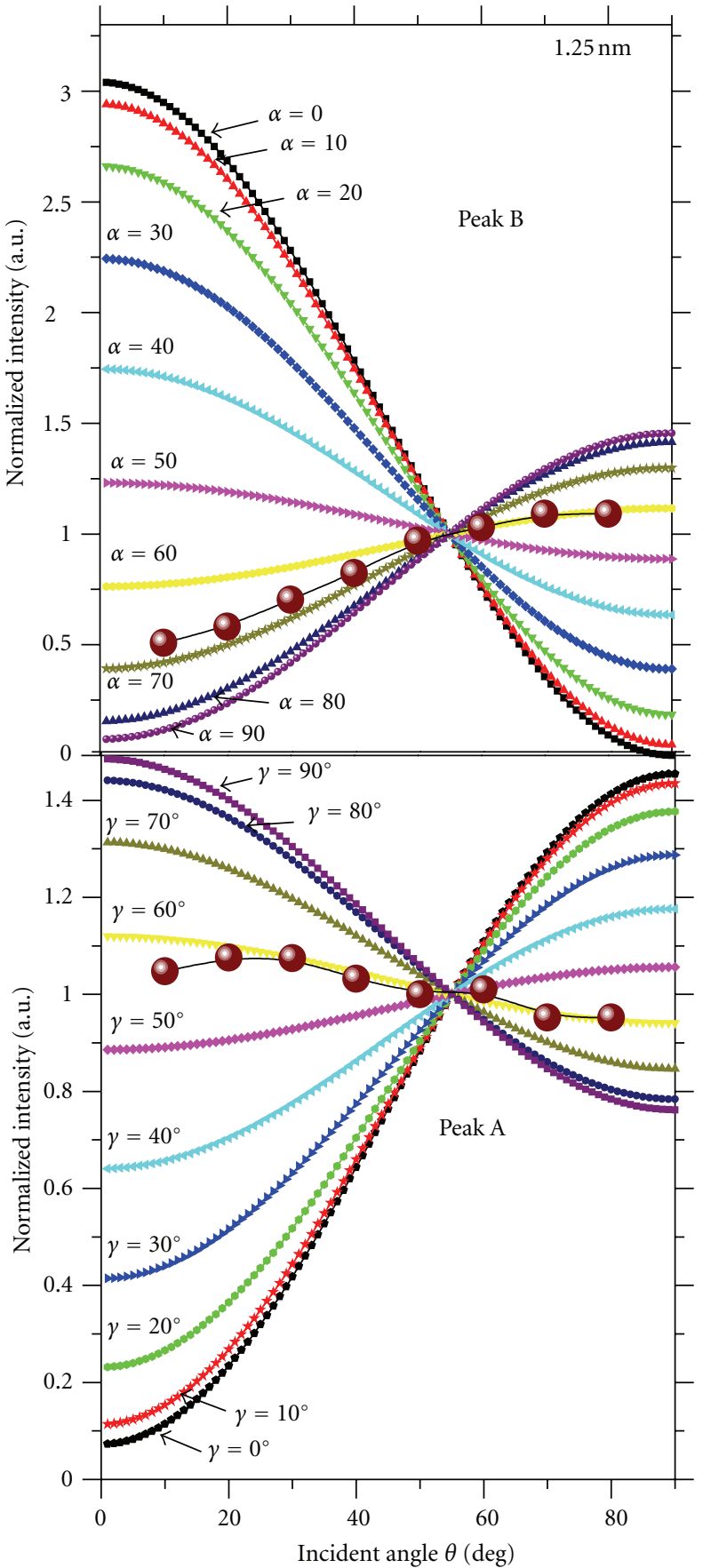

(a)

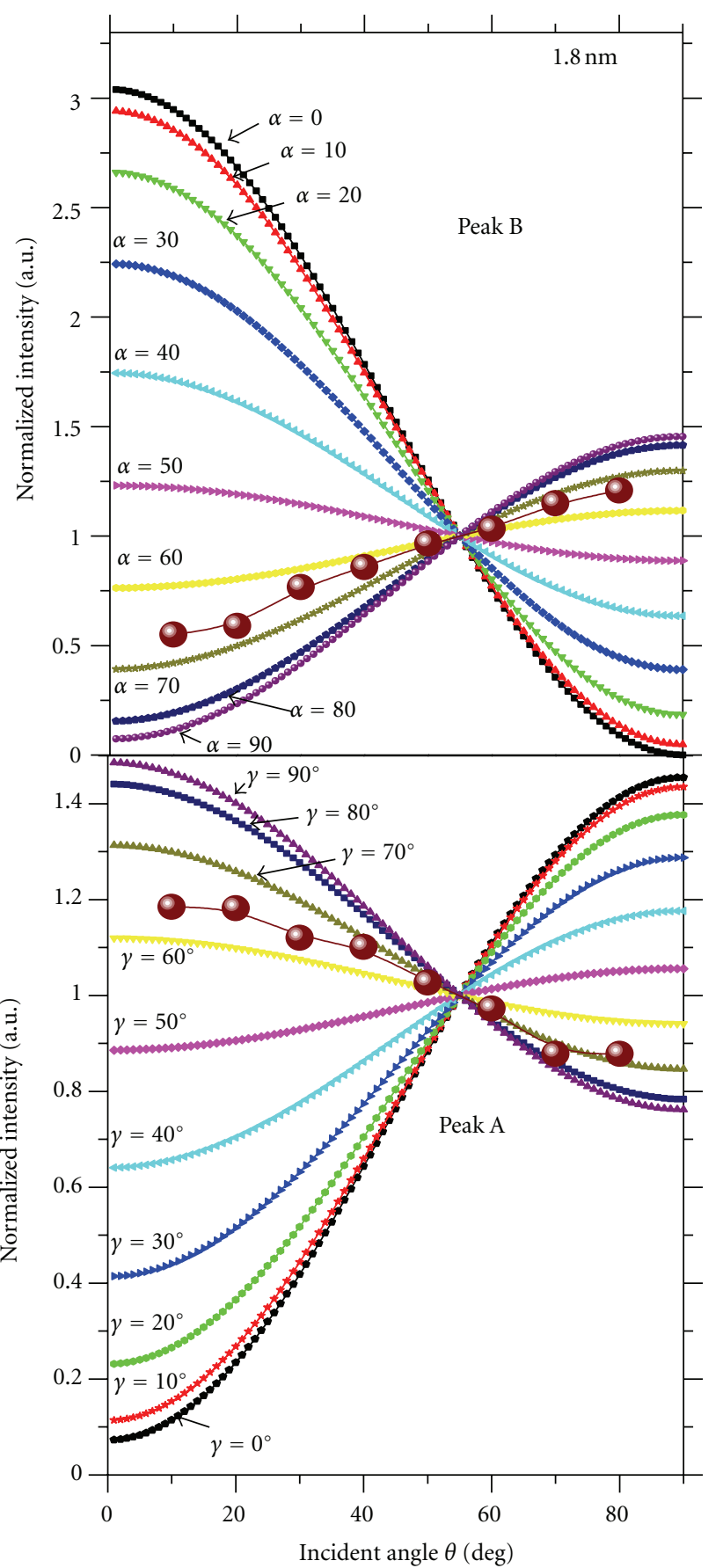

(b)

FIGURE 6: Normalized peak intensity I of the NEXAFS spectra as a function of the incident angle $\theta$ of the SR beam for the samples deposited on HOPG substrate with different thickness: (a) $1.25 \mathrm{~nm}$ and (b) $1.80 \mathrm{~nm}$. The filled circles are the experimental values. The intensities are normalized such that $\mathbf{I}=1$ at $\theta=54.7^{\circ}$ corresponding to the magic angle. The intensities of the peaks A and B were normalized by the total electron yields at $1890 \mathrm{eV}$ for the respective incident angles where no polarization dependences existed. The coloured lines in the upper section of Figure 6 are theoretically calculated values at various $\alpha$ using (4), (5), and (6), while the coloured lines in the lower section of the Figure 6 are those obtained at various $\gamma$ values using (4), (7), and (8) shown in the text.

study which described that the backbones of the PDMS are perpendicular in respect to the ITO surface. The flatlying feature of PDMS observed only on HOPG surface is attributed to the interaction between $\mathrm{CH}$ bonds in PDMS and $\pi$ orbitals in HOPG surface.

\section{Acknowledgments}

The authors like to express their gratitude to staff of the KEKPF for their enough support throughout the experiments. The research has been done with the approval of the Photon 
Factory Program Advisory Committee (the proposal no. PFPAC 2009G553).

\section{References}

[1] R. West, "The polysilane high polymers," Journal of Organometallic Chemistry, vol. 300, no. 1-2, pp. 327-346, 1986.

[2] J. W. Mintmire, "Conformational effects in organopolysilanes: a first-principles approach," Physical Review B, vol. 39, no. 18, pp. 13350-13357, 1989.

[3] K. Seki, H. Ishii, A. Yuyama et al., "Electronic structures of polysilanes and related compounds," Journal of Electron Spectroscopy and Related Phenomena, vol. 78, pp. 403-406, 1996.

[4] J. F. Rabolt, D. Hofer, R. D. Miller, and G. N. Fickes, "Studies of chain conformational kinetics in poly(di-n-alkylsilanes) by spectroscopic methods. 1. Poly(di-n-hexylsilane), poly(din-heptylsilane), and poly(di-n-octylsilane)," Macromolecules, vol. 19, no. 3, pp. 611-616, 1986.

[5] V. M. Hallmark, R. Sooriyakumaran, R. D. Miller, and J. F. Rabolt, "Studies of chain conformational kinetics in poly(din-alkylsilanes) by spectroscopic methods. III. Fourier transform Raman studies of unsymmetric side chain substitution," The Journal of Chemical Physics, vol. 90, no. 4, pp. 2486-2491, 1989.

[6] F. C. Schilling, A. J. Lovinger, J. M. Zeigler, D. D. Davis, and F. A. Bovey, "Solid-state structures and thermochromism of poly(di-n-butylsilylene) and poly(di-n-pentylsilylene)," Macromolecules, vol. 22, no. 7, pp. 3055-3063, 1989.

[7] S. Furukawa and K. I. Takeuchi, "Structure and orientation of polydimethylsilane films," Solid State Communications, vol. 87, no. 10, pp. 931-934, 1993.

[8] S. Furukawa, K. Takeuchi, T. Nomura, T. Yasuda, and M. Tamura, "Estimation of the physical constants of a polydimethylsilane crystal and its evaporated film," Journal of Physics, vol. 5, no. 27, pp. 4729-4736, 1993.

[9] S. Furukawa, M. Obana, T. Nakamine, Y. Shirakawa, A. Sorai, and M. Tamura, "Structure and optical properties of ternary Si:C:H films prepared by evaporation of organo-polysilane," Journal of Physics, vol. 4, no. 22, pp. 5167-5172, 1992.

[10] K. I. Takeuchi, M. Mizoguchi, M. Kira, M. Shimana, S. Furukawa, and M. Tamura, "Orientation of films prepared by the evaporation of poly(dimethyl silane)," Journal of Physics, vol. 6, no. 49, pp. 10705-10712, 1994.

[11] S. Furukawa, "Structure and orientation control of organopolysilanes and their application to electronic devices," Thin Solid Films, vol. 331, no. 1-2, pp. 222-228, 1998.

[12] S. Furukawa and H. Ohta, "Structure and orientation of vacuum-evaporated poly(di-methyl silane) film," Thin Solid Films, vol. 438-439, pp. 48-55, 2003.

[13] M. Gröppel, W. Roth, N. Elbel, and H. von Seggern, "Selfassembly of organopolysilanes on graphite: a scanning tunneling microscopy study," Surface Science, vol. 323, no. 3, pp. 304-310, 1995.

[14] M. A. Mannan, Y. Baba, T. Sekiguchi et al., "Self-ordering of silicon polymer thin film grown on indium tin oxide surface investigated by X-ray absorption spectroscopy," Journal of Electron Spectroscopy and Related Phenomena, vol. 181, no. 23, pp. 242-248, 2010.

[15] H. Konishi, A. Yokoya, H. Shiwaku et al., "Synchrotron radiation beamline to study radioactive materials at the Photon Factory," Nuclear Instruments and Methods in Physics Research A, vol. 372, no. 1-2, pp. 322-332, 1996.
[16] J. H. Scofield, "Theoretical photoionization cross section from 1 to $1500 \mathrm{keV}$," Livermore UCRL 51326, Lawrence Livermore Laboratory, Livermore, Calif, USA, 1973.

[17] M. P. Seah and W. A. Dench, "Quantitative electron spectroscopy of surfaces: a standard data base for electron inelastic mean free paths in solids," Surface and Interface Analysis, vol. 1, no. 1, pp. 2-11, 1979.

[18] Y. Baba, T. Sekiguchi, I. Shimoyama, and K. G. Nath, "Structures of sub-monolayered silicon carbide films," Applied Surface Science, vol. 237, no. 1-4, pp. 176-180, 2004.

[19] K. Sakamoto, D. Kondo, Y. Ushimi et al., “Temperature dependence of the electronic structure of $\mathrm{C}_{60}$ films adsorbed on Si(001)-(2x1) and Si(111)-(7x7) surfaces," Physical Review B, vol. 60, no. 4, pp. 2579-2591, 1999.

[20] T. M. Parrill and Y. W. Chung, "Surface analysis of cubic silicon carbide (001)," Surface Science, vol. 243, no. 1-3, pp. 96-112, 1991.

[21] G. Dufour, F. Rochet, F. C. Stedile et al., "SiC formation by reaction of $\mathrm{Si}(001)$ with acetylene: electronic structure and growth mode," Physical Review B, vol. 56, no. 7, pp. 42664282, 1997.

[22] K. G. Nath, I. Shimoyama, T. Sekiguchi, and Y. Baba, "Chemical-state analysis for low-dimensional Si and Ge films on graphite," Journal of Applied Physics, vol. 94, no. 7, pp. 4583-4588, 2003.

[23] K. G. Nath, I. Shimoyama, T. Sekiguchi, and Y. Baba, "Synchrotron radiation photoabsorption and photoemission spectroscopy for thermal-induced reoriented Si polymer," Journal of Electron Spectroscopy and Related Phenomena, vol. 144-147, pp. 323-326, 2005.

[24] V. R. McCrary, F. Sette, C. T. Chen et al., "Polarization effects in the valence and inner-shell spectra of poly(di-n-hexylsilane)," The Journal of Chemical Physics, vol. 88, no. 9, pp. 5925-5933, 1988.

[25] J. Stöhr, NEXAFS Spectroscopy, vol. 25 of Springer Series in Surface Sciences, Springer, Berlin, Germany, 1996.

[26] J. Deng, Y. Baba, T. Sekiguchi, N. Hirao, and M. Honda, "Effect of substrates on the molecular orientation of silicon phthalocyanine dichloride thin films," Journal of Physics Condensed Matter, vol. 19, no. 19, Article ID 196205, 2007.

[27] M. Nishio, " $\mathrm{CH} / \pi$ hydrogen bonds in crystals," CrystEngComm, vol. 6, pp. 130-158, 2004.

[28] M. Nishio, Y. Umezawa, K. Honda, S. Tsuboyama, and H. Suezawa, " $\mathrm{CH} / \pi$ hydrogen bonds in organic and organometallic chemistry," CrystEngComm, vol. 11, no. 9, pp. 1757-1788, 2009.

[29] M. Nishio, "The $\mathrm{CH} / \pi$ hydrogen bond in chemistry. Conformation, supramolecules, optical resolution and interactions involving carbohydrates," Physical Chemistry Chemical Physics, vol. 13, no. 31, pp. 13873-13900, 2011.

[30] M. Linares, P. Iavicoli, K. Psychogyiopoulou et al., "Chiral expression at the Solid-Liquid interface: a joint experimental and theoretical study of the self-assembly of chiral porphyrins on graphite," Langmuir, vol. 24, no. 17, pp. 9566-9574, 2008. 

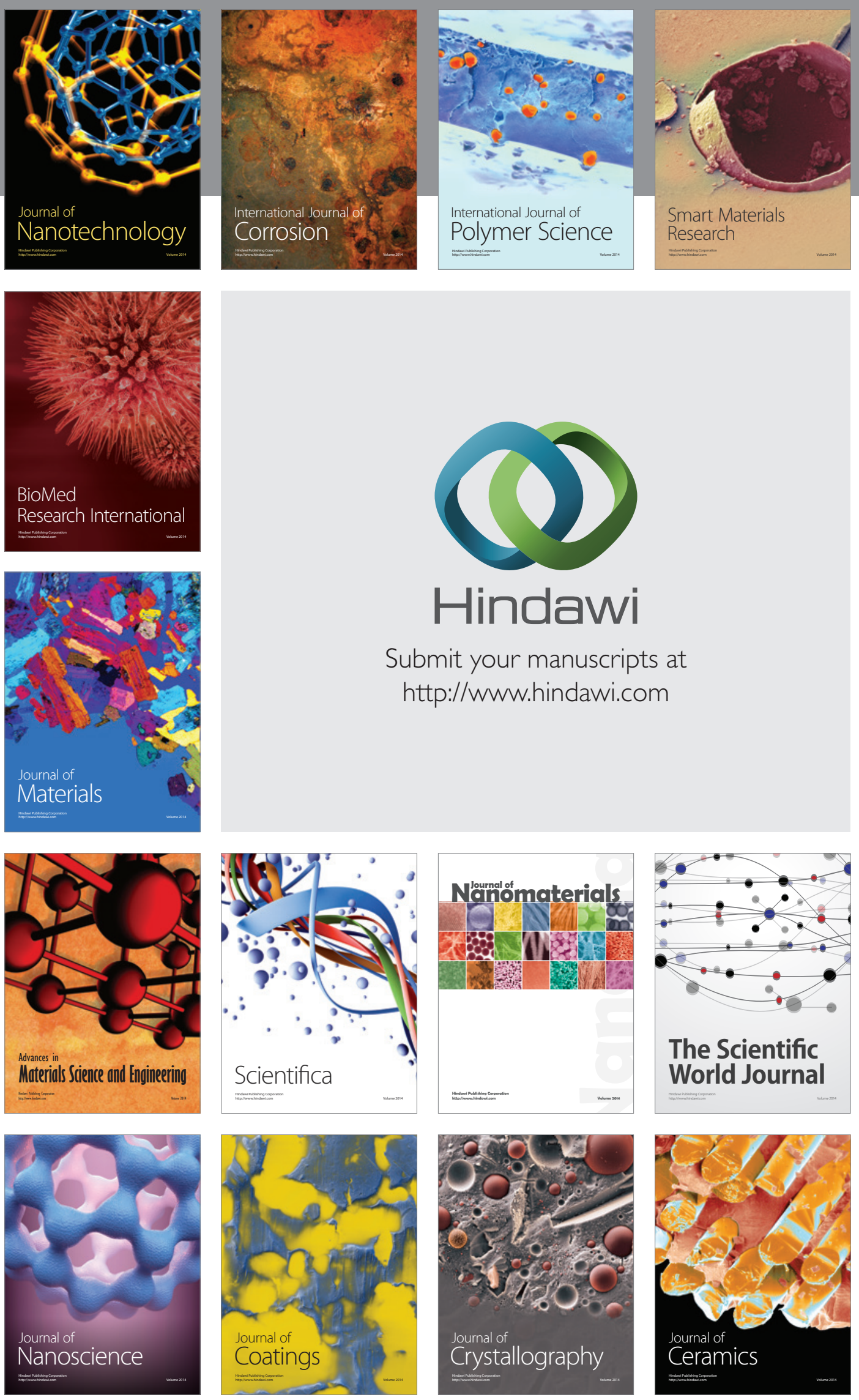

The Scientific World Journal

Submit your manuscripts at

http://www.hindawi.com

\section{World Journal}

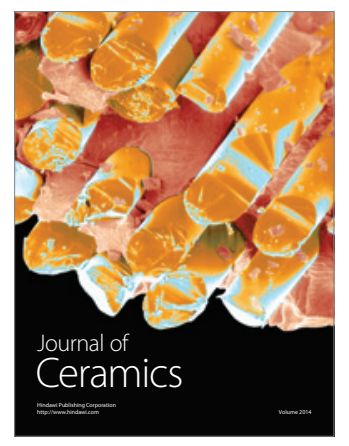

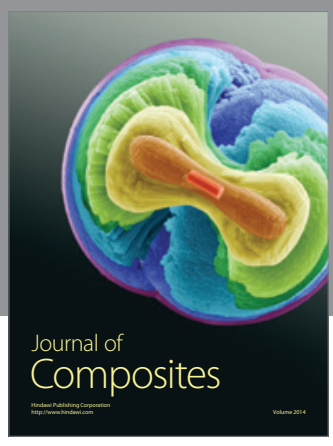
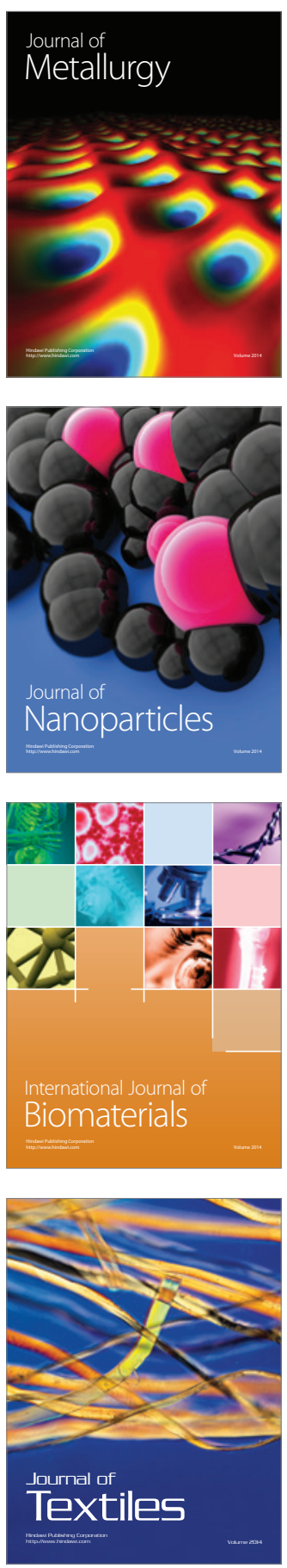